\title{
mTOR signaling in the arcuate nucleus of the hypothalamus mediates the anorectic action of estradiol
}

\author{
Ismael González-García1,2, Pablo B Martínez de Morentin¹,2, Ánxela Estévez-Salguero1,2, Cristina Contreras1,2, \\ Amparo Romero-Picó1,2, Johan Fernø ${ }^{3,4}$, Rubén Nogueiras ${ }^{1,2}$, Carlos Diéguez,2, Manuel Tena-Sempere ${ }^{5,6,7}$, \\ Sulay Tovar1,2 and Miguel López ${ }^{1,2}$
}

1Department of Physiology, CiMUS, University of Santiago de Compostela-Instituto de Investigación Sanitaria, Santiago de Compostela, Spain ${ }^{2}$ CIBER Fisiopatología de la Obesidad y Nutrición (CIBERobn), Santiago de Compostela, Spain ${ }^{3}$ Hormone Laboratory, Haukeland University Hospital, Bergen, Norway

${ }^{4}$ KG Jebsen Center for Diabetes Research, Department of Clinical Science, University of Bergen, Bergen, Norway ${ }^{5}$ Department of Cell Biology, Physiology and Immunology, University of Córdoba, Córdoba, Spain ${ }^{6}$ Instituto Maimónides de Investigación Biomédica (IMIBIC)/Hospital Reina Sofía, Córdoba, Spain 7FiDiPro Program, University of Turku, Turku, Finland

Correspondence should be addressed to S Tovar or M López: sulay.tovar@usc.es or m.lopez@usc.es

\section{Abstract}

Current evidence suggests that estradiol (E2), the main ovarian steroid, modulates energy balance by regulating both feeding and energy expenditure at the central level, through the energy sensor AMP-activated protein kinase (AMPK). We hypothesized that the hypothalamic mechanistic target of rapamycin (mTOR) pathway, a well-established nutrient sensor and modulator of appetite and puberty, could also mediate the anorectic effect of E2. Our data showed that ovariectomy (OVX) elicited a marked downregulation of the mTOR signaling in the arcuate nucleus of the hypothalamus (ARC), an effect that was reversed by either E2 replacement or central estrogen receptor alpha (ER $\alpha$ ) agonism. The significance of this molecular signaling was given by the genetic inactivation of $\mathrm{S} 6$ kinase B1 (S6K1, a key downstream mTOR effector) in the ARC, which prevented the E2-induced hypophagia and weight loss. Overall, these data indicate that E2 induces hypophagia through modulation of mTOR pathway in the ARC.

Key Words
- estradiol
- hypothalamus
- food intake
mTOR
- obesity

Journal of Endocrinology (2018) 238, 177-186

\section{Introduction}

Besides the regulation of the reproductive function, estrogens have a key role in the central regulation of the energy homeostasis including both modulation of feeding behavior and energy expenditure (Mauvais-Jarvis et al. 2013, López \& Tena-Sempere 2015, 2016, 2017, Palmer \& Clegg 2015). Increased life expectancy implies that many women will live an increasing number of years in a state of ovarian insufficiency. This leads to a steady surge in obesity incidence reaching a staggering figure of greater than 70\% in women older than 60 years (Flegal et al. 2010). Although the interrelationship between estrogen deficiency and obesity was the subject of some discussion, pooled data derived 107 trials showed that hormonereplacement therapy in menopausal patients led to reduced abdominal obesity, insulin resistance, new-onset diabetes, lipids, blood pressure, adhesion molecules and procoagulant factors in women without diabetes and reduced insulin resistance, as well as fasting glucose in 
women with diabetes (Salpeter et al. 2006), thus providing a cause-effect relationship between estrogen deficiency, obesity and metabolic complications.

From a mechanistic point of view, studies carried out in rodents showed that reduced levels of estradiol (E2) after ovariectomy (OVX) are associated with hyperphagia and decreased energy expenditure, leading to weight gain (Martínez de Morentin et al. 2014a, 2015). Moreover, variations in the magnitude of meals and body weight occur in rats throughout the estrous cycle, in parallel with changes in endogenous E2 levels (Blaustein \& Wade 1976, Tritos et al. 2004, Mauvais-Jarvis et al. 2013, Martínez de Morentin et al. 2014a, López \& Tena-Sempere 2015, 2017). The functional relevance of these data is supported by the fact that central administration of E2 elicits profound anorectic, catabolic and weight-reducing effects (Martínez de Morentin et al. 2014a, 2015).

At the central level, estrogen receptor alpha and beta $(E R \alpha$ and $E R \beta)$ are expressed in several hypothalamic nuclei with key roles in the regulation of energy balance, such as the arcuate (ARC), paraventricular (PVH) and ventromedial (VMH) (Simerly et al. 1990, Simonian \& Herbison 1997, Voisin et al. 1997, Osterlund et al. 1998, Merchenthaler et al. 2004). Recent evidence has shown that $\mathrm{E} 2$ has a nucleus-specific action in the hypothalamus to modulate energy homeostasis, particularly within the ARC and the VMH. Thus, while most of the actions of estrogens on food intake take place through ER $\alpha$ in the ARC, its effect on energy expenditure is conducted through ER $\alpha$ in the VMH (Xu et al. 2011, Martínez de Morentin et al. 2014a, 2015). The molecular mechanisms mediating the effect of central estrogens are not totally understood, but recent data have demonstrated that E2 acting on ER $\alpha$ inhibits hypothalamic AMP-activated protein kinase (AMPK) and that genetic activation of this enzyme within the ARC reverses the anorectic action of E2 (Martínez de Morentin et al. 2014a).

The mechanistic target of rapamycin (mTOR) is an evolutionarily conserved serine-threonine kinase that acts as a cellular sensor of changes in growth factors, nutrients and oxygen (Wang \& Proud 2009, Laplante \& Sabatini 2012, Martinez de Morentin et al. 2014b). mTOR phosphorylates and modulates the activity of the serine/ threonine ribosomal protein S6 kinase B1 (S6K1). In turn, S6K1 phosphorylates and activates $\mathrm{S} 6$, a ribosomal protein involved in protein translation (Wang \& Proud 2009, Laplante \& Sabatini 2012, Martinez de Morentin et al. $2014 b$ ). Specifically, hypothalamic mTOR signaling plays a key role in modulating energy balance by responding to nutrient availability and the hormonal milieu
(Cota et al. 2006, 2008, Blouet et al. 2008, Mori et al. 2009, Martins et al. 2012, Varela et al. 2012). Furthermore, a link between mTOR and the gonadal axis has been reported. Specifically, hypothalamic mTOR controls puberty onset and gonadotropin secretion by regulation of Kiss 1 (Roa et al. 2009, Roa \& Tena-Sempere 2010). This evidence indicates that mTOR signaling could contribute to the functional coupling between energy balance and gonadal activation and function. However, despite this evidence, it remains unclear whether mTOR might mediate the central actions of estrogens on food intake. The aim of this study was to assess whether the anorectic actions of E2 are mediated by specific modulation of mTOR signaling in the ARC.

\section{Materials and methods}

\section{Animals}

Adult female Sprague-Dawley rats (250-300g; Animalario General USC, Santiago de Compostela, Spain) were used for the experiments. The experiments were performed in agreement with 'International Law on Animal Experimentation' and were approved by the USC Ethical Committee (Project ID 15010/14/006). The animals were housed with an artificial 12-h light (08:00-20:00)/12-h darkness cycle, under controlled temperature and humidity conditions and allowed free access to standard laboratory chow and tap water. For all the procedures, except during the washout period after OVX, the animals were caged individually.

\section{Ovariectomy}

Rats were bilaterally OVX or sham-operated, in which each ovary was exposed but not tied or dissected, as previously described (Martínez de Morentin et al. 2014a, 2015, Skrede et al. 2017). All treatments (central or peripheral) on OVX rats were carried out 2 weeks after surgery to ensure a total washout of ovarian hormones, as previously reported (Martínez de Morentin et al. 2014a, 2015, Skrede et al. 2017).

\section{Determination of estrous cycle}

Female rats were monitored for estrous cycle by daily vaginal cytology, and only rats with at least two consecutive regular 4-day estrous cycles were used in expression analyses, as previously reported (Martínez de Morentin et al. 2014a).

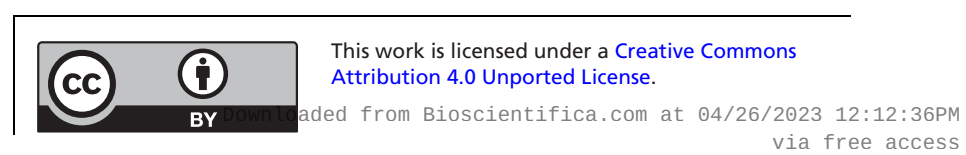




\section{Peripheral and central treatments}

For the experiments with E2 replacement, OVX rats received a daily subcutaneous (SC) injection of estradiol benzoate $(2 \mu \mathrm{g}$ dissolved in $100 \mu \mathrm{L}$ of sesame oil; both from Sigma) or vehicle $(100 \mu \mathrm{L}$ of sesame oil; control rats) during 5-11 days (Vigo et al. 2007, Roa et al. 2009, Martínez de Morentin et al. 2014a, 2015, Skrede et al. 2017).

For the central treatments, intracerebroventricular (ICV) cannulae were stereotaxically implanted under ketamine/xylazine anesthesia, as previously described (Varela et al. 2012, Martínez de Morentin et al. 2014a, 2015, Martínez-Sánchez et al. 2017). Animals were individually housed and used for experimentation 4 days later. For the central estrogen receptor agonists setting, OVX rats received one daily injection of the selective ER $\alpha$ agonist 4,4',4"-(4-propyl-[1H]-pyrazole-1,3,5-triyl) trisphenol (PPT, $5 \mathrm{nmol}$ dissolved in $5 \mu \mathrm{L}$ of DMSO; TOCRIS Bioscience, Bristol, UK) or vehicle ( $5 \mu \mathrm{L}$ of DMSO; control rats) for 5 days (Sanchez-Criado et al. 2004, 2006, Roa et al. 2008, Martínez de Morentin et al. 2014a, 2015). For the central leucine (Leu) experiments, OVX rats received one daily injection of Leu $(10 \mathrm{nmol}$ dissolved in $5 \mu \mathrm{L}$ of saline) (Laeger et al. 2014) or vehicle ( $5 \mu \mathrm{L}$ of saline) for 6 days.

\section{Stereotaxic microinjection}

Rats treated with vehicle or E2 were placed in a stereotaxic frame (David Kopf Instruments, Tujunga, CA, USA) under ketamine/xylazine anesthesia. The ARC was targeted using a 25-gauge needle (Hamilton, Reno, $\mathrm{NV}$, USA). The ARC injections were bilaterally directed to the following stereotaxic coordinates: $-2.8 \mathrm{~mm}$ posterior (one injection was performed in each ARC), $\pm 0.3 \mathrm{~mm}$ lateral to bregma and $10.2 \mathrm{~mm}$ dorso-ventral, as previously reported (Varela et al. 2012, Contreras et al. 2014, Martínez de Morentin et al. 2014a, MartínezSánchez et al. 2017). Adenoviral vectors (SignaGen, Rockville, MD, USA) encoding green fluorescence protein (GFP, used as control), S6K1 dominant negative (S6K1-DN; at $10^{10} \mathrm{pfu} / \mu \mathrm{L}$ ) or $\mathrm{S} 6 \mathrm{~K} 1$ constitutively active (S6K1-CA; at $5 \times 10^{10} \mathrm{pfu} / \mu \mathrm{L}$ ) isoforms, were delivered at a rate of $200 \mathrm{~nL} / \mathrm{min}$ for $5 \mathrm{~min}(1 \mu \mathrm{L} /$ injection site $)$ as previously reported (Varela et al. 2012, Contreras et al. 2014, Martínez de Morentin et al. 2014a, MartínezSánchez et al. 2017). The adenoviral and E2 treatments started at the same time. Direct detection of GFP fluorescence was performed after perfusion of the rats

$$
\text { http://joe.endocrinology-journals.org }
$$

and detected with a fluorescence microscope Olympus IX51 at $4 \times$ augmentation.

\section{Sample processing}

Rats were killed by cervical dislocation and decapitation. From each animal, the ARC was collected and immediately homogenized on ice to preserve phosphorylated protein levels. Those samples and the serum were stored at $-80^{\circ} \mathrm{C}$ until further processing. Dissection of the ARC was performed by micro-punch procedure under the microscope, as previously described (Varela et al. 2012, Contreras et al. 2014, Martínez de Morentin et al. 2014a, Martínez-Sánchez et al. 2017). The specificity of the ARC dissections was confirmed by analyzing the protein levels of the specific marker proopiomelanocortin (POMC; data not shown).

\section{Hormone measurements}

Circulating E2 levels were determined using a commercial ultra-sensitive RIA kit (Beckman Coulter, Brea, CA, USA). The sensitivity of the assay was $2.2 \mathrm{pg} / \mathrm{mL}$, and the intraand inter-assay CVs were $8.9 \%$ and $12.2 \%$, respectively.

\section{Western blotting}

ARC protein lysates were subjected to SDS-PAGE, electrotransferred on a PVDF membrane and probed with the following antibodies: mTOR, pmTOR Ser2448, S6K1, pS6K1 Thr389, S6, pS6 Ser235/236 (Cell Signalling, Danvers, MA, USA), and $\beta$-actin (Abcam, Cambridge, UK) as previously described (Varela et al. 2012, Martínez de Morentin et al. 2014a, 2015, Martínez-Sánchez et al. 2017). Values were expressed relative to $\beta$-actin protein levels. Autoradiographic films were scanned and the bands signal was quantified by densitometry using ImageJ-1.33 software (NIH, Bethesda, MD, USA). Representative images for all proteins are shown and each protein was corrected by its own internal $\beta$-actin control. In the gel images, all the bands for each picture come always from the same gel, but they may be spliced for clarity, as indicated in the figure legends.

\section{Statistical analysis}

Data are expressed as mean \pm s.e.m. Protein data are expressed relative (\%) to control (Sham, OVX, vehicle or GFP treated) rats. Statistical significance is determined by Student $t$ test when two groups is compared or ANOVA

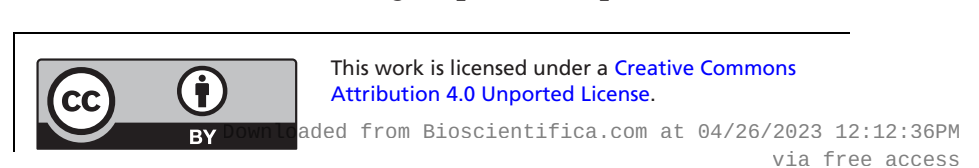


and post hoc Bonferroni test when more than two groups are compared. $P<0.05$ is considered significant. The number of animals used in each experimental setting and analysis are specified in each figure legend.

\section{Results}

\section{Lack of ovarian function decreases mTOR signaling in the ARC}

OVX rats gained significantly more weight and developed marked hyperphagia (Fig. 1A and B). OVX rats showed the expected decrease in circulating E2 levels (Sham: $21.75 \pm 2.68 \mathrm{pg} / \mathrm{mL}$ vs OVX: $9.35 \pm 0.49 \mathrm{pg} / \mathrm{mL} ; P<0.001$; $n=9$ rats/group) confirming the efficiency of the OVX procedure. Our data showed that OVX induced a marked inactivation of mTOR signaling, as demonstrated by decreased protein levels in the ARC of phosphorylated (active) mTOR (pmTOR) at Ser2448, and its downstream targets, namely, pS6K1 at Thr389 and pS6 at Ser235/236
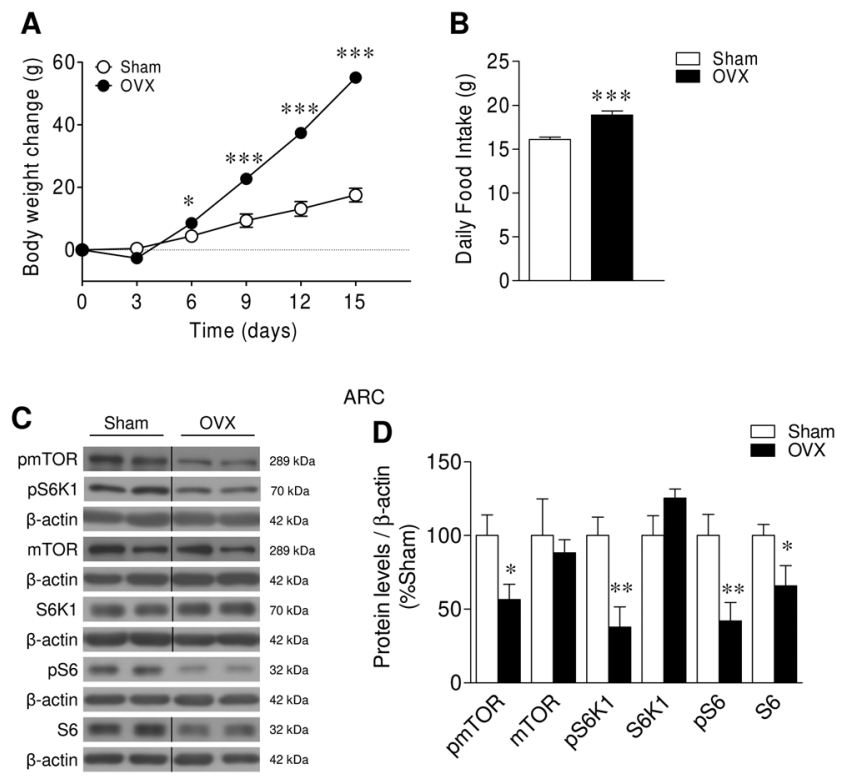

Figure 1

Effect of ovariectomy on energy balance and mTOR pathway within the ARC. (A) Body weight change, (B) daily food intake, (C) representative Western blot auto-radiographic images and (D) ARC protein levels of mTOR pathway of Sham rats or OVX rats. $n=30-32$ animals per group for body weight and food intake data; $n=7$ animals per group for Western blot data. All data are expressed as mean \pm S.E.M. * ** and $* * * P<0.05,0.01$ and 0.001 vs Sham. For the Western blot analysis, representative images for all proteins are shown. In the gel images, all the bands for each picture come always from the same gel, but they may be spliced for clarity; in such case, this is depicted as vertical black lines.
(Fig. 1C and D). These data suggested that ovarian function regulated mTOR signaling in the ARC.

\section{Estradiol increases mTOR signaling in the ARC}

To gain more insight in the effect of ovarian function on mTOR, we analyzed the effects of E2 replacement on mTOR signaling in the ARC of OVX rats. Our data showed that $\mathrm{E} 2$ administration to OVX rats induced weight loss and reduced feeding (Fig. 2A and B), associated with activation of the ARC MTOR pathway, demonstrated by elevated levels of pmTOR, pS6K1 and pS6 in that nucleus (Fig. 2C and D). To further explore the physiological relevance of our findings, we investigated the modulation of mTOR pathway of rats in proestrous, a stage of the cycle with elevated E2 levels (Martínez de Morentin et al. 2014a, 2015, Skrede et al. 2017). Our data showed that when compared with OVX animals, rats at proestrous exhibited a higher activation of mTOR signaling in the ARC (Supplementary Fig. 1A, see section

A
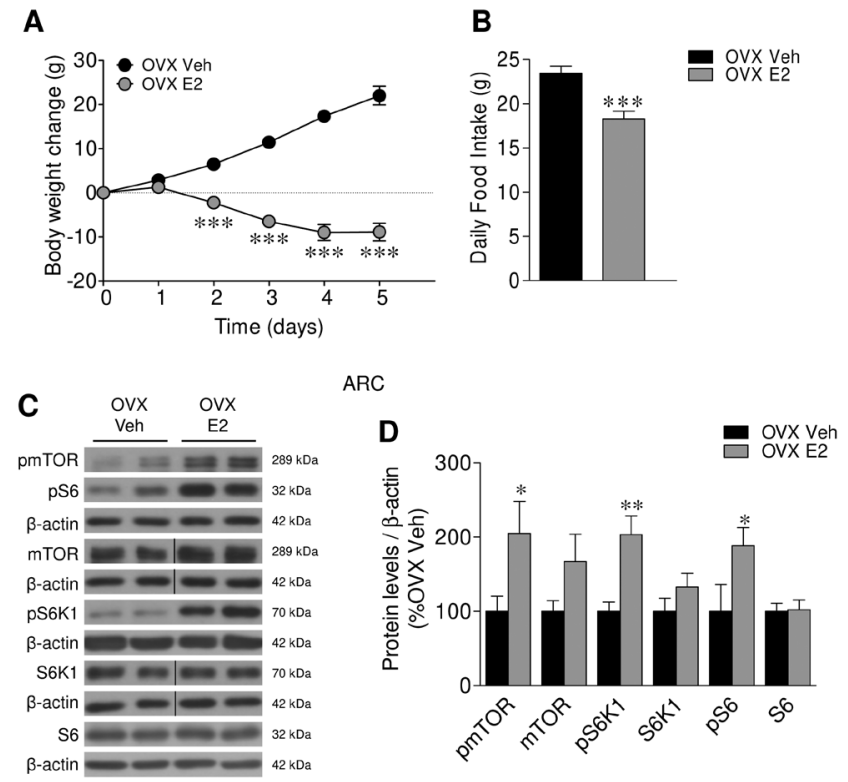

Figure 2

Effect of E2 replacement on energy balance and $\mathrm{mTOR}$ pathway within the ARC in OVX rats. (A) Body weight change, (B) daily food intake, (C) representative Western blot auto-radiographic images and (D) ARC protein levels of mTOR pathway of OVX rats SC treated with vehicle or E2. $n=8-10$ animals per group for body weight and food intake data; $n=6-10$ animals per group for Western blot data. All data are expressed as mean \pm S.E.M. ${ }^{*}, * *$ and $* * * P<0.05,0.01$ and 0.001 vs OVX vehicle. For the Western blot analysis, representative images for all proteins are shown. In the gel images, all the bands for each picture come always from the same gel, but they may be spliced for clarity; in such case, this is depicted as vertical black lines.

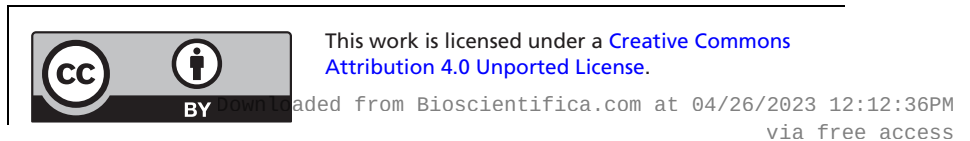


on supplementary data given at the end of this article). This evidence suggested that endogenous E2 levels were likely physiological regulators on hypothalamic mTOR.

\section{ER $\alpha$ agonism increases mTOR signaling in the ARC}

Compelling evidence has demonstrated that the anorectic effect of E2 is mediated by ER $\alpha$ in the ARC (Xu et al. 2011); therefore, we aimed to investigate whether the effect of E2 on mTOR signaling was mediated by this receptor. ICV administration of the specific ER $\alpha$ agonist, PPT (Sanchez-Criado et al. 2004, 2006, Roa et al. 2008, Martínez de Morentin et al. 2014a, 2015), to OVX rats mostly recapitulated the effects of $\mathrm{E} 2$ by eliciting weight loss and anorexia (Fig. 3A and B), with concomitant activation of the mTOR pathway in the ARC (Fig. 3C and D), being the slight differences observed likely related to the route of administration (SC E2 vs ICV PPT). Overall, these data demonstrated that the anorectic effect of E2 in the ARC was associated to increased mTOR signaling in this hypothalamic nucleus, likely via the ER $\alpha$ receptor.
A

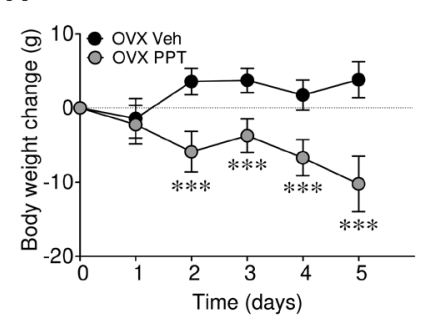

B

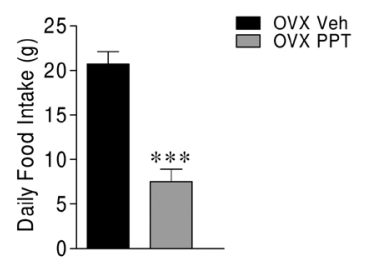

ARC

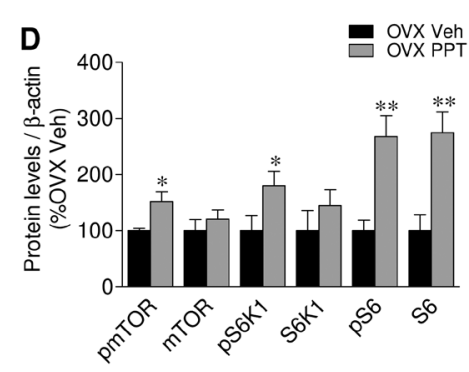

Figure 3

Effect of central PPT on energy balance and mTOR pathway within the $A R C$ in OVX rats. (A) Body weight change, (B) daily food intake, (C) representative Western blot auto-radiographic images and (D) ARC protein levels of mTOR pathway of OVX rats ICV treated with vehicle or PPT. $n=7-12$ animals per group for body weight and food intake data; $n=7$ animals per group for Western blot data. All data are expressed as mean \pm S.E.M. ${ }^{*}, * *$ and $* * * P<0.05,0.01$ and 0.001 vs OVX vehicle. For the Western blot analysis, representative images for all proteins are shown In the gel images, all the bands for each picture come always from the same gel, but they may be spliced for clarity; in such case, this is depicted as vertical black lines.
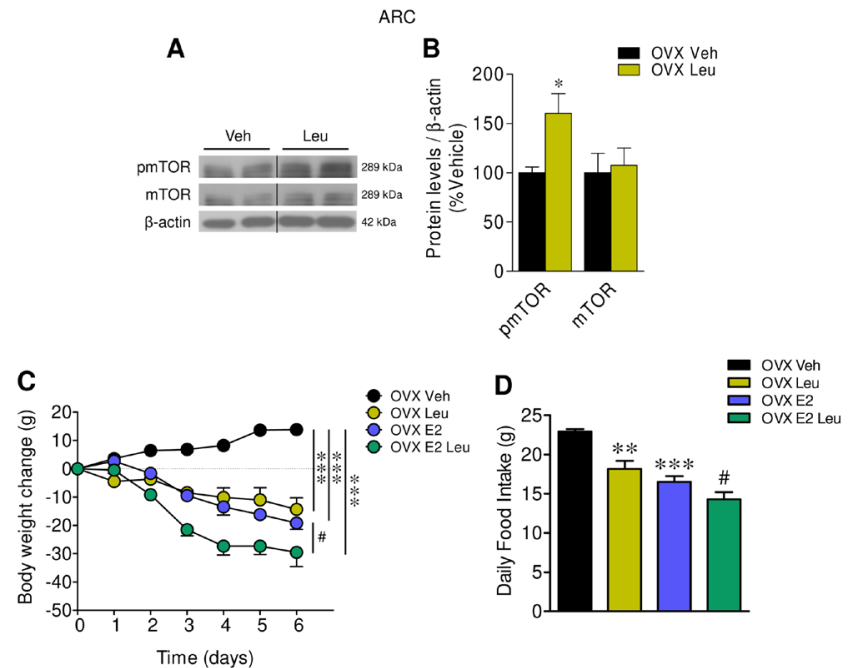

Figure 4

Effect of central Leu and E2 on energy balance in OVX rats.

(A) Representative Western blot autoradiographic images and

(B) densitometry measures of protein levels of pmTOR and mTOR in the ARC of OVX rats ICV treated with vehicle or Leu. (C) Body weight change and (D) daily food intake of OVX rats ICV treated with vehicle or Leu and/or SC treated with vehicle or E2; $n=6$ animals per group for body weight, food intake and Western blot data. All data are expressed as mean \pm S.E.M. * ${ }^{* *}$ and $* * * P<0.05, P<0.01$ and 0.001 vs OVX vehicle; ${ }^{\#} P<0.05$ vs OVX E2. For the Western blot analysis, representative images for all proteins are shown. In the gel images, all the bands for each picture come always from the same gel, but they may be spliced for clarity; in such case, this is depicted as vertical black lines.

\section{Central activation of mTOR with leucine recapitulates the effect of $\mathrm{E} 2$}

Given that E2- and PPT-induced anorexia in association to increased mTOR signaling in the ARC of OVX rats, we next aimed to investigate whether activation of this pathway impacted feeding in OVX rats. Our data showed that central administration of Leu, a well-established activator of mTOR (Cota et al. 2006, Laeger et al. 2014) increased the levels of pmTOR in the ARC (Fig. 4A and B), and decreased body weight $(P<0.001 ; F=23.67)$ and food intake $(P<0.001 ; F=18.88)$ in OVX rats in a similar magnitude to E2 (Fig. 4C and D).

\section{Inhibition of S6K1 in the ARC reversed the anorectic effect of E2}

To further investigate the role of mTOR signaling on the anorectic effect of E2, we targeted S6K1, a direct downstream target of mTOR by using adenoviruses encoding either S6K1-CA or S6K1-DN or control adenovirus expressing GFP in the ARC. Injection in the ARC was assessed by visualization of GFP expression (Fig. 5A) and the efficiency of the treatment by assessing

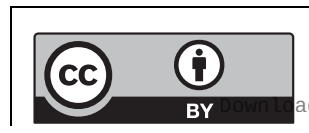

This work is licensed under a Creative Commons Attribution 4.0 Unported License. 
A

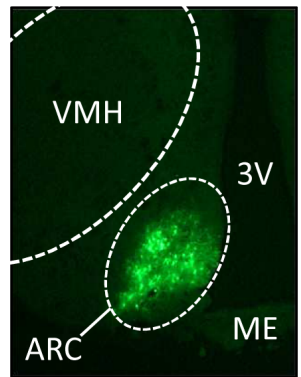

B

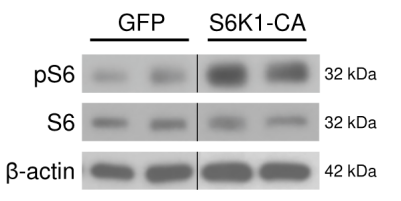

ARC

D
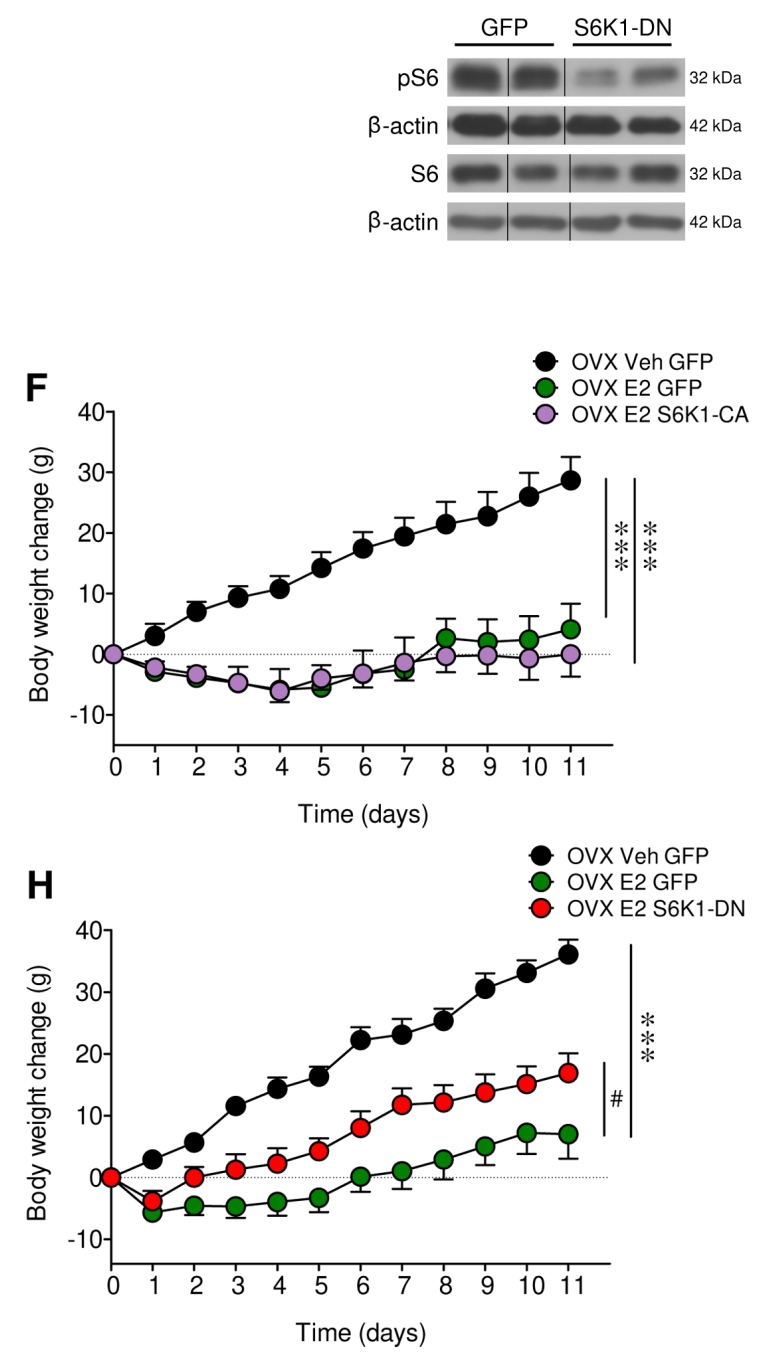

ARC
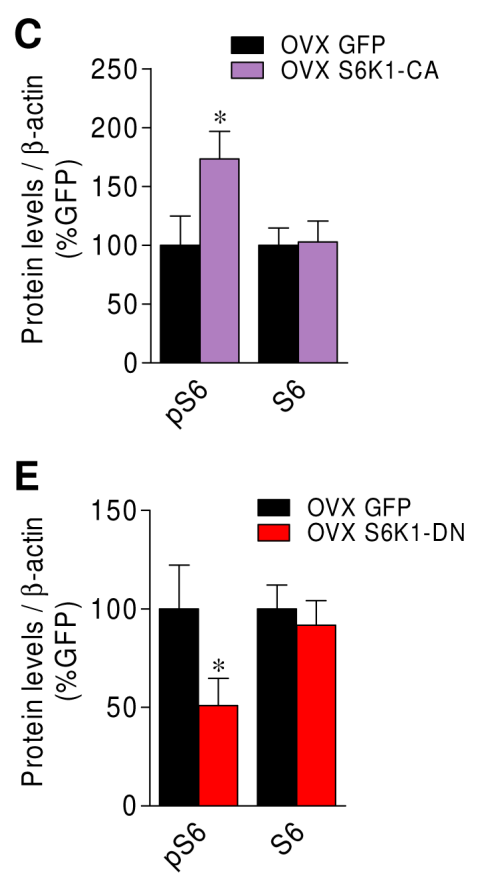

G

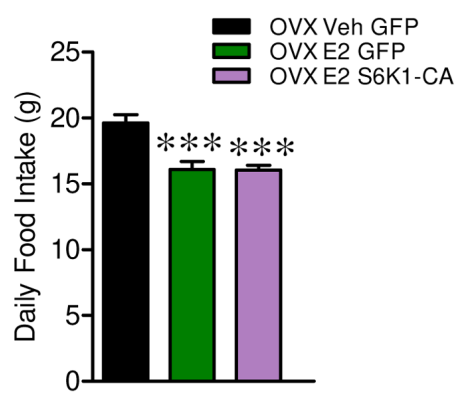

Figure 5

Effect of activation and down-regulation of mTOR pathway on the central actions of E2 on energy balance in OVX rats. (A) Direct fluorescence of GFP, $(B$ and $D)$ representative Western blot auto-radiographic images and (C and E) protein levels of pS6 and S6 in the ARC of OVX rats stereotaxically treated in the ARC with adenoviruses encoding GFP, S6K1-CA or S6K1-DN. (F) Body weight change and (G) daily food intake of OVX rats stereotaxically treated in the ARC with adenoviruses encoding GFP or S6K1-CA and SC treated with vehicle or E2. (H) Body weight change and (I) daily food intake of OVX rats stereotaxically treated in the ARC with adenoviruses encoding GFP or S6K1-DN and SC treated with vehicle or E2. $n=8-11$ animals per group for body weight and food intake data; $n=7$ animals per group for Western blot data. All data are expressed as mean \pm s.E.M. * and $* * * P<0.05$ and 0.001 vs GFP or OVX Veh GFP; $\# P<0.05$ vs OVX E2 GFP. For the Western blot analysis, representative images for all proteins are shown. In the gel images, all the bands for each picture come always from the same gel, but they may be spliced for clarity; in such case, this is depicted as vertical black lines.

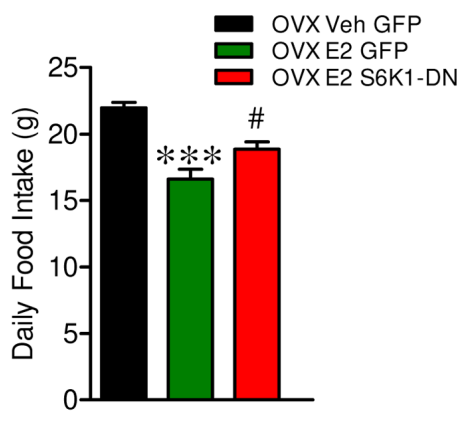


the protein levels of pS6, the downstream target of S6K1. S6K1-CA treatment increased, while S6K1-DN decreased, pS6 levels in the ARC, when compared with GFP controls (Fig. 5B, C, D and E). While administration of S6K1-CA in the ARC of E2-treated OVX rats decreased body weight $(P<0.001 ; F=15.95)$ and reduced feeding $(P<0.01 ; F=6.50)$ in a similar magnitude to E2 (Fig. 5F and G), S6K1-DN ameliorated the effect of simultaneously given E2 by increased food intake $(P<0.001 ; F=15.98)$ and therefore body mass $(P<0.001 ; F=20.33)$ (Fig. $5 \mathrm{H}$ and I). No changes in body weight were detected when vehicle-treated OVX rats were administered with S6K1-CA or S6K1-DN adenoviruses (Supplementary Fig. 2A and B). Overall, these data indicate that the anorectic effect of E2 is, in part, mediated by stimulation of mTOR signaling in the ARC.

\section{Discussion}

Current evidence has demonstrated that E2's anorectic effect is mediated by ER $\alpha$ signaling in the ARC (Xu et al. 2011, Martínez de Morentin et al. 2014a, 2015). However, the molecular mechanism explaining this effect remains unclear. Here, we show for the first time that mTOR signaling in the ARC is modulated by E2, and specifically by ER $\alpha$ agonism, an effect that mediates the anorectic actions of this estrogen.

Over the last decade, it has become clear that besides the classical neuropeptide networks, key molecular pathways regulate energy balance in the hypothalamus. Among them, energy sensors, such as AMPK (Kahn et al. 2005, López et al. 2016, López 2017) and nutrient sensors, such as mTOR (Cota et al. 2006, Blouet et al. 2008, Varela et al. 2012, Martinez de Morentin et al. 2014b) play a major role. We have recently documented the role of AMPK in the central actions of E2. Our data suggest that AMPK mediates the effects of E2 on energy balance in a dual manner; food intake is regulated via effects on AMPK in the ARC, whereas modulation of AMPK action in the VMH controls energy expenditure through sympathetic regulation of the brown fat (Martínez de Morentin et al. 2014a, 2015).

Whether mTOR signaling plays a role in the anorectic actions of central E2 is currently unknown, but the idea is supported by data showing that AMPK and mTOR pathways interact in the hypothalamus to modulate energy balance (Dagon et al. 2012, Varela et al. 2012) and also by the fact that the mTOR route mediates the effects on feeding of other peripheral signals such as thyroid hormones and ghrelin (Martins et al. 2012, Varela et al. 2012). Moreover, it is known that mTOR acts in the hypothalamus regulating the hypothalamus-pituitarygonadal axis (HPG) (Roa et al. 2009). All this evidence led us to investigate the possible connection of the hypothalamic mTOR pathway with E2's anorectic action.

Our data show that mTOR signaling is modulated by E2 acting at the central level, as demonstrated by the fact that E2 administration reversed the OVX-induced decrease in mTOR signaling in the ARC. One possible constraint of our study is the use of OVX rats, which are probably the most 'classical' model for the study of ovarian steroid actions (Martínez de Morentin et al. 2014a, 2015, Skrede et al. 2017). In this context, the major strength of this model, namely the lack of ovarian estrogens, it is also a limitation, which makes difficult to extrapolate the conclusions to a physiological context (the ovarianintact, cycling female), perhaps apart from aging-related decline in ovarian function. To overrule this limitation, we analyzed the mTOR pathway in rats at the proestrous stage of the cycle, when the E2 levels are maximal (Martínez de Morentin et al. 2014a, 2015). Our data showed that mTOR signaling in the ARC is activated in proestrous when compared with OVX rats, suggesting that the mTOR pathway in the ARC is physiologically regulated by ovarian steroid milieu. Of course, other regulators of hypothalamic mTOR cannot be ruled out. For example, it is known that the increased adiposity that follows OVX is associated with increased leptinemia and insulinemia (Mauvais-Jarvis et al. 2013). Considering that both leptin and insulin are major modulators of hypothalamic mTOR (Dagon et al. 2012, Martinez de Morentin et al. 2014b), thus, the possible development of central leptin and/or insulin resistance could be a contributing factor.

One interesting fact is that the effect of $\mathrm{E} 2$ on mTOR signaling is mediated by $\mathrm{ER} \alpha$ in this nucleus, since administration of the specific ER $\alpha$ agonist, PPT, mostly recapitulates (with slight differences in feeding, possible due to the protocol of administration: SC E2 vs ICV PPT) this action. This is of importance, because ER $\alpha$ is the primary ER isoform to modulate the anorectic properties of E2 at the hypothalamic level (Xu et al. 2011, Martinez de Morentin et al. 2015). Therefore, we hypothesized that hypophagia and subsequent weight loss after E2 or PPT administration might be mediated by specific modulation of mTOR signaling in the ARC. Our results show that either pharmacological activation of mTOR signaling following central administration of Leu or genetic activation of the downstream protein S6K1 with specifically delivery of S6K1-CA adenoviruses in the ARC prevented the 
OVX-induced hyperphagia and body weight gain. Importantly, the specific inhibition of the S6K1 in the ARC with S6K1-DN isoforms, partially blunted the anorectic effect of E2. These data demonstrate that the central actions of E2 on energy balance are at least partially mediated by the selective modulation of mTOR pathway through ER $\alpha$ and that this effect is placed in the ARC.

The molecular relevance of this evidence is intriguing. AMPK and mTOR function as major regulators of cellular metabolism that respond to changes in energy and nutrient status (Martinez de Morentin et al. 2014b, López et al. 2016). Both in vitro and in vivo results have demonstrated that activation of AMPK suppresses mTOR signaling (Bolster et al. 2002, Krause et al. 2002, Kimura et al. 2003, Saha et al. 2010) and also that S6K1 phosphorylates AMPK to mediate leptin's action on feeding (Dagon et al. 2012). Furthermore, AMPK phosphorylates mTOR at Thr2446, inhibiting its function, which in turn decreases S6K1 phosphorylation (Cheng et al. 2004). Therefore, considering that AMPK in the ARC mediates the appetitesuppressive effect of E2 (Martínez de Morentin et al. $2014 a$ ), it would be tempting to speculate that both routes might act in this hypothalamic nucleus to coordinately regulate feeding and energy balance, a hypothesis that warrants further studies. In this sense, it will be critical to identify the ARC neuronal populations mediating that action. The most obvious candidate would be POMC neurons, which have been demonstrated to be critical for mediating E2's anorectic effects (Xu et al. 2011, Martinez de Morentin et al. 2015). However, the well-described actions of E2 on specific glial cell populations, such as astrocytes (Azcoitia et al. 2011, Acaz-Fonseca et al. 2014, 2016), with a known role on energy balance (Garcia-Caceres et al. 2016), and the fact that the used S6K1 adenoviruses can also infect those cells, make astrocytes also interesting candidates to explore in further studies. The molecular underpinnings of that effect are also of interest for investigation. In this sense, the existence of membraneinitiated estrogen signaling effects in POMC neurons, involving for example mitogen-activated protein kinase (MAPK), phosphoinositide 3-kinase (PI3K) and protein kinase C (PKC) (López \& Tena-Sempere 2015, Stincic et al. 2018), which are upstream modulators of mTOR (Martinez de Morentin et al. 2014b, Saxton \& Sabatini 2017), makes them potential candidates to mediate the effects of E2 signaling on mTOR and, subsequently, its anorectic action.

In summary, our study shows that mTOR signaling in the ARC conveys E2's anorectic effect. Our data also describe that hypothalamic mTOR pathway is of importance for understanding and potential treatment of the positive energy balance that characterizes states of estrogen deficiency, such as OVX or menopause.

\section{Supplementary data}

This is linked to the online version of the paper at https://doi.org/10.1530/ JOE-18-0190.

Declaration of interest

The authors declare that there is no conflict of interest that could be perceived as prejudicing the impartiality of the research reported.

\section{Funding}

This work did not receive any specific grant from any funding agency in the public, commercial, or not-for-profit sector.

\section{Authors' contribution statement}

I G-G, P B M M, A E-S and C C performed the in vivo experiments (OVX, peripheral, central and stereotaxic microinjections), the analytical methods (hormone measurements and Western blotting) and collected the data. A R-P validated the S6K1 adenoviruses. I G-G, P B M M, J F, R N, C D, M T-S, $S T$ and $M L$ designed the experiments, analyzed, discussed and interpreted the data. I G-G and M L made the figures. All authors reviewed and edited the manuscript and had final approval of the submitted version. M L developed the hypothesis, wrote the manuscript, coordinated and directed the project and secured funding.

\section{Acknowledgements}

The authors dedicate this work to the bright memory of our colleague, master and friend Enrique Aguilar. The research leading to these results has received funding from Xunta de Galicia (R N: 2015-CP080 and 2016PG057; M L: 2015-CP079), Junta de Andalucía (M T-S: P12-FQM-01943), MINECO co-funded by the FEDER Program of EU (C D: BFU2017-87721; R N: BFU2015-70664R; M T-S: BFU2014-57581-P and PIE14/0005; M L: SAF201571026-R and BFU2015-70454-REDT/Adipoplast). The CiMUS is supported by the Xunta de Galicia (2016-2019, ED431G/05). CIBER Fisiopatología de la Obesidad y Nutrición is an initiative of ISCIII. A E-S is a recipient of a fellowship from MINECO (FPI/BES-2016-077439). The funders had no role in study design, data collection and analysis, decision to publish or preparation of the manuscript.

\section{References}

Acaz-Fonseca E, Sanchez-Gonzalez R, Azcoitia I, Arevalo MA \& GarciaSegura LM 2014 Role of astrocytes in the neuroprotective actions of 17 beta-estradiol and selective estrogen receptor modulators. Molecular and Cellular Endocrinology 389 48-57. (https://doi.org/10.1016/j. mce.2014.01.009)

Acaz-Fonseca E, Avila-Rodriguez M, Garcia-Segura LM \& Barreto GE 2016 Regulation of astroglia by gonadal steroid hormones under physiological and pathological conditions. Progress in Neurobiology 144 5-26. (https://doi.org/10.1016/j.pneurobio.2016.06.002)

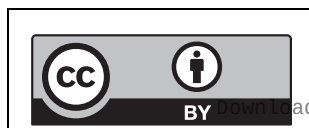

This work is licensed under a Creative Commons Attribution 4.0 Unported License. 
Azcoitia I, Arevalo MA, De Nicola AF \& Garcia-Segura LM 2011 Neuroprotective actions of estradiol revisited. Trends in Endocrinology and Metabolism 22 467-473. (https://doi.org/10.1016/j. tem.2011.08.002)

Blaustein JD \& Wade GN 1976 Ovarian influences on the meal patterns of female rats. Physiology and Behavior 17 201-208. (https://doi. org/10.1016/0031-9384(76)90064-0)

Blouet C, Ono H \& Schwartz GJ 2008 Mediobasal hypothalamic p70 S6 kinase 1 modulates the control of energy homeostasis. Cell Metabolism 8 459-467. (https://doi.org/10.1016/j.cmet.2008.10.004)

Bolster DR, Crozier SJ, Kimball SR \& Jefferson LS 2002 AMP-activated protein kinase suppresses protein synthesis in rat skeletal muscle through down-regulated mammalian target of rapamycin (mTOR) signaling. Journal of Biological Chemistry 277 23977-23980. (https:// doi.org/10.1074/jbc.C200171200)

Cheng SW, Fryer LG, Carling D \& Shepherd PR 2004 Thr2446 is a novel mammalian target of rapamycin (mTOR) phosphorylation site regulated by nutrient status. Journal of Biological Chemistry 279 15719-15722. (https://doi.org/10.1074/jbc.C300534200)

Contreras C, González-García I, Martínez-Sánchez N, Seoane-Collazo P, Jacas J, Morgan DA, Serra D, Gallego R, González F, Casals N, et al. 2014 Central ceramide-induced hypothalamic lipotoxicity and ER stress regulate energy balance. Cell Reports 9 366-377. (https://doi. org/10.1016/j.celrep.2014.08.057)

Cota D, Proulx K, Smith KA, Kozma SC, Thomas G, Woods SC \& Seeley RJ 2006 Hypothalamic mTOR signaling regulates food intake. Science 312 927-930. (https://doi.org/10.1126/science.1124147)

Cota D, Matter EK, Woods SC \& Seeley RJ 2008 The role of hypothalamic mammalian target of rapamycin complex 1 signaling in dietinduced obesity. Journal of Neuroscience 28 7202-7208. (https://doi. org/10.1523/JNEUROSCI.1389-08.2008)

Dagon Y, Hur E, Zheng B, Wellenstein K, Cantley LC \& Kahn BB 2012 p70S6 kinase phosphorylates AMPK on serine 491 to mediate leptin's effect on food intake. Cell Metabolism 16 104-112. (https://doi. org/10.1016/j.cmet.2012.05.010)

Flegal KM, Carroll MD, Ogden CL \& Curtin LR 2010 Prevalence and trends in obesity among US adults, 1999-2008. JAMA 303 235-241. (https://doi.org/10.1001/jama.2009.2014)

Garcia-Caceres C, Quarta C, Varela L, Gao Y, Gruber T, Legutko B, Jastroch M, Johansson P, Ninkovic J, Yi CX, et al. 2016 Astrocytic insulin signaling couples brain glucose uptake with nutrient availability. Cell 166 867-880. (https://doi.org/10.1016/j. cell.2016.07.028)

Kahn BB, Alquier T, Carling D \& Hardie DG 2005 AMP-activated protein kinase: ancient energy gauge provides clues to modern understanding of metabolism. Cell Metabolism 1 15-25. (https://doi.org/10.1016/j. cmet.2004.12.003)

Kimura N, Tokunaga C, Dalal S, Richardson C, Yoshino K, Hara K, Kemp BE, Witters LA, Mimura O \& Yonezawa K 2003 A possible linkage between AMP-activated protein kinase (AMPK) and mammalian target of rapamycin (mTOR) signalling pathway. Genes Cells 8 65-79. (https://doi.org/10.1046/j.1365-2443.2003.00615.x)

Krause U, Bertrand L \& Hue L 2002 Control of p70 ribosomal protein S6 kinase and acetyl-CoA carboxylase by AMP-activated protein kinase and protein phosphatases in isolated hepatocytes. European Journal of Biochemistry 269 3751-3759. (https://doi.org/10.1046/j.14321033.2002.03074.x)

Laeger T, Reed SD, Henagan TM, Fernandez DH, Taghavi M, Addington A, Munzberg H, Martin RJ, Hutson SM \& Morrison CD 2014 Leucine acts in the brain to suppress food intake but does not function as a physiological signal of low dietary protein. American Journal of Physiology: Regulatory, Integrative and Comparative Physiology $\mathbf{3 0 7}$ R310-R320. (https://doi.org/10.1152/ajpcell.00192.2014)

Laplante M \& Sabatini DM 2012 mTOR signaling in growth control and disease. Cell 149 274-293. (https://doi.org/10.1016/j. cell.2012.03.017)
López M 2017 EJE PRIZE 2017: Hypothalamic AMPK: a golden target against obesity? European Journal of Endocrinology 176 R235-R246. (https://doi.org/10.1530/EJE-16-0927)

López M \& Tena-Sempere M 2015 Estrogens and the control of energy homeostasis: a brain perspective. Trends in Endocrinology and Metabolism 26 411-421. (https://doi.org/10.1016/j.tem.2015.06.003)

Lopez M \& Tena-Sempere M 2016 Estradiol and brown fat. Best Practice and Research: Clinical Endocrinology and Metabolism 30 527-536. (https://doi.org/10.1016/j.beem.2016.08.004)

López M \& Tena-Sempere M 2017 Estradiol effects on hypothalamic AMPK and BAT thermogenesis: a gateway for obesity treatment? Pharmacology and Therapeutics 178 109-122. (https://doi. org/10.1016/j.pharmthera.2017.03.014)

López M, Nogueiras R, Tena-Sempere M \& Dieguez C 2016 Hypothalamic AMPK: a canonical regulator of whole-body energy balance. Nature Reviews Endocrinology 12 421-432. (https://doi.org/10.1038/ nrendo.2016.67)

Martínez de Morentin PB, Gónzalez-García I, Martins L, Lage R, Fernández-Mallo D, Martínez-Sánchez N, Ruíz-Pino F, Liu J, Morgan DA, Pinilla L, et al. 2014a Estradiol regulates brown adipose tissue thermogenesis via hypothalamic AMPK. Cell Metabolism 20 41-53. (https://doi.org/10.1016/j.cmet.2014.03.031)

Martinez de Morentin PB, Martinez-Sanchez N, Roa J, Ferno J, Nogueiras R, Tena-Sempere M, Dieguez C \& Lopez M $2014 b$ Hypothalamic mTOR: the rookie energy sensor. Current Molecular Medicine 14 3-21. (https://doi.org/10.2174/15665240136661311181 03706)

Martinez de Morentin PB, Lage R, Gonzalez-Garcia I, Ruiz-Pino F, Martins L, Fernandez-Mallo D, Gallego R, Ferno J, Senaris R, Saha AK, et al. 2015 Pregnancy induces resistance to the anorectic effect of hypothalamic malonyl-CoA and the thermogenic effect of hypothalamic AMPK inhibition in female rats. Endocrinology 156 947-960. (https://doi.org/10.1210/en.2014-1611)

Martínez-Sánchez N, Seoane-Collazo P, Contreras C, Varela L, Villarroya J, Rial-Pensado E, Buqué X, Aurrekoetxea I, Delgado T, VázquezMartínez R, et al. 2017 Hypothalamic AMPK-ER stress-JNK1 axis mediates the central actions of thyroid hormones on energy balance. Cell Metabolism 26 212-229. (https://doi.org/10.1016/j. cmet.2017.06.014)

Martins L, Fernette B, Novelle MG, Vázquez MJ, Tena-Sempere M, Nogueiras R, López M \& Diéguez C 2012 Hypothalamic mTOR signaling mediates the orexigenic action of ghrelin. PLOS ONE 7 e46923. (https://doi.org/10.1371/journal.pone.0046923)

Mauvais-Jarvis F, Clegg DJ \& Hevener AL 2013 The role of estrogens in control of energy balance and glucose homeostasis. Endocrine Reviews 34 309-38. (https://doi.org/10.1210/er.2012-1055)

Merchenthaler I, Lane MV, Numan S \& Dellovade TL 2004 Distribution of estrogen receptor alpha and beta in the mouse central nervous system: in vivo autoradiographic and immunocytochemical analyses. Journal of Comparative Neurology 473 270-291. (https://doi. org/10.1002/cne.20128)

Mori H, Inoki K, Munzberg H, Opland D, Faouzi M, Villanueva EC, Ikenoue T, Kwiatkowski D, MacDougald OA, Myers MG Jr, et al. 2009 Critical role for hypothalamic mTOR activity in energy balance. Cell Metabolism 9 362-374. (https://doi.org/10.1016/j.cmet.2009.03.005)

Osterlund M, Kuiper GG, Gustafsson JA \& Hurd YL 1998 Differential distribution and regulation of estrogen receptor-alpha and -beta mRNA within the female rat brain. Brain Research Molecular Brain Research 54 175-180. (https://doi.org/10.1016/S0169328X(97)00351-3)

Palmer BF \& Clegg DJ 2015 The sexual dimorphism of obesity. Molecular and Cellular Endocrinology 402 113-119. (https://doi.org/10.1016/j. mce.2014.11.029)

Roa J \& Tena-Sempere M 2010 Energy balance and puberty onset: emerging role of central mTOR signaling. Trends in Endocrinology and Metabolism 21 519-528. (https://doi.org/10.1016/j.tem.2010.05.003)

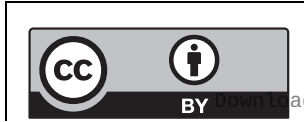

This work is licensed under a Creative Commons Attribution 4.0 Unported License. 
Roa J, Vigo E, Castellano JM, Gaytan F, Navarro VM, Aguilar E, Dijcks FA, Ederveen AG, Pinilla L, van Noort PI, et al. 2008 Opposite roles of estrogen receptor (ER)-alpha and ERbeta in the modulation of luteinizing hormone responses to kisspeptin in the female rat: implications for the generation of the preovulatory surge. Endocrinology 149 1627-1637. (https://doi.org/10.1210/en.2007-1540)

Roa J, García-Galiano D, Varela L, Sánchez-Garrido MA, Pineda R, Castellano JM, Ruíz-Pino F, Romero M, Aguilar E, López M, et al. 2009 The mammalian target of rapamycin as novel central regulator of puberty onset via modulation of hypothalamic Kiss1 system. Endocrinology 150 5016-5026. (https://doi.org/10.1210/en.2009-0096)

Saha AK, Xu XJ, Lawson E, Deoliveira R, Brandon AE, Kraegen EW \& Ruderman NB 2010 Downregulation of AMPK accompanies leucineand glucose-induced increases in protein synthesis and insulin resistance in rat skeletal muscle. Diabetes 59 2426-2434. (https://doi. org/10.2337/db09-1870)

Salpeter SR, Walsh JM, Ormiston TM, Greyber E, Buckley NS \& Salpeter EE 2006 Meta-analysis: effect of hormone-replacement therapy on components of the metabolic syndrome in postmenopausal women. Diabetes, Obesity and Metabolism 8 538-554. (https://doi.org/10.1111/ j.1463-1326.2005.00545.x)

Sanchez-Criado JE, Martin de las MJ, Bellido C, Tena-Sempere M, Aguilar R \& Blanco A 2004 Biological role of pituitary estrogen receptors ERalpha and ERbeta on progesterone receptor expression and action and on gonadotropin and prolactin secretion in the rat. Neuroendocrinology 79 247-258. (https://doi.org/10.1159/000079100)

Sanchez-Criado JE, de Las Mulas JM, Bellido C, Navarro VM, Aguilar R, Garrido-Gracia JC, Malagon MM, Tena-Sempere M \& Blanco A 2006 Gonadotropin-secreting cells in ovariectomized rats treated with different oestrogen receptor ligands: a modulatory role for ERbeta in the gonadotrope? Journal of Endocrinology 188 167-177. (https://doi. $\operatorname{org} / 10.1677 /$ joe.1.06377)

Saxton RA \& Sabatini DM 2017 mTOR signaling in growth, metabolism, and disease. Cell 168 960-976. (https://doi.org/10.1016/j. cell.2017.02.004)

Simerly RB, Chang C, Muramatsu M \& Swanson LW 1990 Distribution of androgen and estrogen receptor mRNA-containing cells in the rat brain: an in situ hybridization study. Journal of Comparative Neurology 294 76-95. (https://doi.org/10.1002/cne.902940107)
Simonian SX \& Herbison AE 1997 Differential expression of estrogen receptor alpha and beta immunoreactivity by oxytocin neurons of rat paraventricular nucleus. Journal of Neuroendocrinology 9 803-806. (https://doi.org/10.1046/j.1365-2826.1997.00659.x)

Skrede S, Gonzalez-Garcia I, Martins L, Berge RK, Nogueiras R, TenaSempere M, Mellgren G, Steen VM, Lopez M \& Ferno J 2017 Lack of ovarian secretions reverts the anabolic action of olanzapine in female rats. International Journal of Neuropsychopharmacology 20 1005-1012. (https://doi.org/10.1093/ijnp/pyx073)

Stincic TL, Ronnekleiv OK \& Kelly MJ 2018 Diverse actions of estradiol on anorexigenic and orexigenic hypothalamic arcuate neurons. Hormones and Behavior [epub]. (https://doi.org/10.1016/j. yhbeh.2018.04.001)

Tritos NA, Segal-Lieberman G, Vezeridis PS \& Maratos-Flier E 2004 Estradiol-induced anorexia is independent of leptin and melaninconcentrating hormone. Obesity Research 12 716-724. (https://doi. org/10.1038/oby.2004.84)

Varela L, Martínez-Sánchez N., Gallego R, Vázquez MJ, Roa J, Gándara M., Schoenmakers E, Nogueiras R, Chatterjee K, Tena-Sempere M, et al. 2012 Hypothalamic mTOR pathway mediates thyroid hormoneinduced hyperphagia in hyperthyroidism. Journal of Pathology 227 209-222. (https://doi.org/10.1002/path.3984)

Vigo E, Roa J, Lopez M, Castellano JM, Fernandez-Fernandez R, Navarro VM, Pineda R, Aguilar E, Dieguez C, Pinilla L, et al. 2007 Neuromedin $\mathrm{s}$ as novel putative regulator of luteinizing hormone secretion. Endocrinology 148 813-823. (https://doi.org/10.1210/ en.2006-0636)

Voisin DL, Simonian SX \& Herbison AE 1997 Identification of estrogen receptor-containing neurons projecting to the rat supraoptic nucleus. Neuroscience 78 215-228. (https://doi.org/10.1016/S03064522(96)00551-9)

Wang X \& Proud CG 2009 Nutrient control of TORC1, a cell-cycle regulator. Trends in Cell Biology 19 260-267. (https://doi.org/10.1016/j. tcb.2009.03.005)

Xu Y, Nedungadi TP, Zhu L, Sobhani N, Irani BG, Davis KE, Zhang X, Zou F, Gent LM, Hahner LD, et al. 2011 Distinct hypothalamic neurons mediate estrogenic effects on energy homeostasis and reproduction. Cell Metabolism 14 453-465. (https://doi.org/10.1016/j. cmet.2011.08.009)

Received in final form 11 June 2018

Accepted 18 June 2018

Accepted Preprint published online 18 June 2018

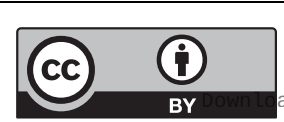

This work is licensed under a Creative Commons Attribution 4.0 Unported License. 